\title{
Stereotactic radiosurgery may contribute to overall survival for patients with recurrent head and neck carcinoma
}

\author{
Koji Kawaguchi*1, Kengo Sato², Akihisa Horie1, Susumu Iketani³, Hiroyuki Yamada1', Yasunori Nakatani³, Junichi Sato \\ and Yoshiki Hamada'
}

\begin{abstract}
Background: The aim of this study is to examine the effect of stereotactic radiosurgery (SRS) in the treatment of advanced, recurrent lesions for head and neck carcinoma both with and without lymph node involvement.

Methods: Between April 2006 and July 2007, 22 patients (mean age 67 years) with advanced, recurrent head and neck carcinoma were treated with stereotactic radiosurgery. All of the patients except one had biopsy confirmed disease prior to stereotactic radiosurgery. Patients included 3 rT2, 8 rT3, and 9 rT4; 8 of the patients had lymph node metastases. Marginal SRS doses were 20-42 Gy delivered in two to five fractions. Starting one month after SRS, all patients received S-1 oral chemotherapy for one year.

Results: At an overall median follow-up of 24 months (range, 4-39 months), for the 14 locally recurrent patients without lymph node metastases, 9 patients (64.3\%) had a complete response (CR), 1 patient (7.1\%) had a partial response (PR), 1 patient (7.1\%) had stable disease (SD), and 3 patients (21.4\%) had progressive disease (PD). For the 8 patients with lymph node metastases, 1 patient with a single retropharyngeal (12.5\%) had CR; the remaining 7 patients (87.5\%) all progressed. Nine patients have died from their cancer. The overall actuarial 2-year survival for the patients with and without lymph node metastases is $12.5 \%$ and $78.6 \%$, respectively.
\end{abstract}

Conclusions: These results show the benefit of stereotactic radiosurgery salvage treatment for advanced, recurrent lesions, without lymph node metastases in previously irradiated head and neck cancer.

\section{Background}

The majority of head and neck region squamous cell carcinomas present at an advanced stage and are treated with a combined-modality approach that often includes surgery, radiation therapy, and chemotherapy. Despite such aggressive approaches, advanced squamous cell carcinoma of the head and neck tends to recur locoregionally and, thus, presents a significant clinical challenge [13]. Surgery and/or conventional chemoradiotherapy salvage is difficult in advanced, recurrent lesions of head and neck carcinoma given the proximity of critical organs. Similarly, radical treatment for wide recurrent lesions is limited by overall radiation doses for the body

\footnotetext{
* Correspondence: kawaguchi-k@tsurumi-u.ac.jp

1 Department of Oral and Maxillofacial Surgery, Tsurumi University, School of Dental Medicine. 2-1-3 Tsurumi, Tsurumi-ku, Yokohama, 230-8501, Japan Full list of author information is available at the end of the article
}

and the possibility of severe post-operative dysfunction. In cases where further surgery is not feasible, reirradiation offers the potential to gain locoregional control and achieve palliation [4].

Several studies have confirmed the feasibility of reirradiation of recurrent head and neck tumors, with curative intent using external beam radiation therapy (EBRT) [58]. More recently, stereotactic radiosurgery (SRS) has been employed in the treatment of head and neck cancers both in primary cases $[9,10]$ and in recurrent cases [1115]. The CyberKnife (Accuray Incorporated, Sunnyvale, California, USA) is a frameless robotic radiosurgery system that has been utilized by numerous clinicians around the world to treat intracranial and extracranial tumors $[9,13,16,17]$. The CyberKnife image-guided radiosurgical system can deliver isocentric or non-isocentric beams with high precision and high dose conformity [18]. These 
abilities are especially important when treating irregularly shaped tumors, or those in difficult locations close to critical structures, as is the case with many of the patients with advanced, recurrent head and neck cancer. Here, we report on the tumor response and overall survival of stereotactic radiosurgery treatment using the CyberKnife for advanced, recurrent head and neck carcinoma lesions both with and without lymph node metastases.

\section{Methods}

Between April 2006 and July 2007, 22 patients with advanced, recurrent head and neck squamous cell carcinoma were treated at the Yokohama CyberKnife Center, Yokohama, Japan. All patients included in this study completed an informed consent form. Patients were examined using PET-CT, MRI, and Ultrasound. Prior to radiation therapy, biopsies were performed for all patients, except for one for whom the recurrence was located deep in their temple muscle. For that patient, diagnosis was determined based upon the high level of uptake observed on PET scan. We assumed that this was a metastasis from the original squamous cell carcinoma that was located on the floor of their oral cavity. Hence, we denoted this patient as M1.

Prior to stereotactic radiosurgery, 21 patients $(95.5 \%)$ had surgical treatment and 13 of those patients received post-operative chemo-radiotherapy. The remaining patient (4.7\%) received prior irradiation, but neither prior surgery nor chemotherapy. The prior conventional irradiation was delivered as a wide field with doses of 40-65 Gy delivered in 1.5-2.0 Gy daily fractions. In all cases, the SRS was delivered within the prior irradiation field. The overall interval between prior treatment and SRS treatment was a median 11 months (range, 4-21 months). For those patients that received prior irradiation, SRS treatment occurred a median 11 months (range, 4-21 months) later. For those that received surgery alone, SRS treatment occurred a median 14 months (range, 7-26 months) later. Table 1 provides an overview of the patient characteristics.

Stereotactic radiosurgery was delivered with the CyberKnife (Accuray Inc., Sunnyvale, USA), an X-band linear accelerator with an overall system targeting error of less than $1 \mathrm{~mm}[18,19]$. The lightweight linear accelerator is capable of irradiating the target from 120 different directions using image-guidance based on a treatment plan created using a CT volume [13,20,21]. To assist with treatment planning, the $\mathrm{CT}$ image was also fused with an MRI or PET-CT image as applicable. During both treatment planning and delivery, patients were imaged while wearing a custom-made mouthpiece, to immobilize the moving parts of the mouth, and a thermoplastic mask fixed to the treatment couch, to minimize head move-
Table 1: Patient characteristics.

\begin{tabular}{|c|c|}
\hline Sex & Number (\%) \\
\hline Male & $8(36 \%)$ \\
\hline Female & $14(64 \%)$ \\
\hline \multicolumn{2}{|l|}{ Age, years } \\
\hline Median (range) & $67(42-91)$ \\
\hline \multicolumn{2}{|l|}{ Initial treatment } \\
\hline Surgery alone & $8(36 \%)$ \\
\hline $\begin{array}{r}\text { Surgery + Post-operative } \\
\text { Chemo-Radiation }\end{array}$ & $13(59 \%)$ \\
\hline Radiation & $1(5 \%)$ \\
\hline
\end{tabular}

Prior radiation dose (Gy)

Total (daily) dose range

$40-65(1.5-2)$

Histology

Squamous cell carcinoma

$21(95.5 \%)$

M1 case

$1(4.5 \%)$

Regions of recurrence or

metastases

\begin{tabular}{rc}
\hline Tongue & $7(31.8 \%)$ \\
\hline Mandible & $5(22.7 \%)$ \\
\hline Maxilla & $3(13.6 \%)$ \\
\hline Soft palate & $3(13.6 \%)$ \\
\hline Limited with lymph node & $2(9.1 \%)$ \\
\hline Temporal muscle & $1(4.5 \%)$ \\
\hline
\end{tabular}

Clinical Stage

rTON1M0

$1(4.5 \%)$ 
Table 1: Patient characteristics. (Continued)

\begin{tabular}{lc}
\hline rTONOM1 & $1(4.5 \%)$ \\
\hline rT2NOMO & $3(13.6 \%)$ \\
\hline rT3N0M0 & $5(22.7 \%)$ \\
\hline rT3N1M0 & $2(9.1 \%)$ \\
\hline rT3N2MO & $1(4.5 \%)$ \\
\hline rT4N0MO & $5(22.7 \%)$ \\
\hline rT4N1M0 & $2(9.1 \%)$ \\
\hline rT4N2MO & $2(9.1 \%)$ \\
\hline
\end{tabular}

ment. Treatment was administered depending upon the configuration and volume of the tumor as determined by the treating radiation oncologist, neurosurgeon and oral and maxillofacial surgeon. Dose constraints were applied to nearby critical structures based upon the total dose and fractionation scheme. Specifically, the dose to brain stem, optic nerve, optic chiasm, retina, and spinal cord were each limited to 21-25 Gy; the dose to the carotid artery, esophagus, and larynx were each limited to 30-35 Gy; and the dose to the eye lens was limited to 7-10 Gy. The prescribed dose of radiation was administered to the clinical target volume without the addition of any margin, corresponding to the $80-85 \%$ isodose contour. In the cases of lymph nodes metastases, the lymph nodes were treated. In general, the dose to those lesions which previously received irradiation was reduced by $20 \%$ from that of those patients for which no prior radiation was delivered to the lesion. In those cases where the PTV was more than $30 \mathrm{cc}$ the dose was reduced by $30 \%$. Overall, patients were treated with a median marginal dose of 33.73 Gy (range, 20-42 Gy) in two to five fractions with treatment delivered over consecutive days. The median gross lesion diameter was $36.63 \mathrm{~mm}$ (range, 15.21-58.65 $\mathrm{mm}$ ). The median irradiated volume was $24.5 \mathrm{~cm}^{3}$ (range, $3.4-74.4 \mathrm{~cm}^{3}$ ). Table 2 provides a summary of the treatment details.

In addition to stereotactic radiosurgery, a low dose of oral chemotherapy S-1 (oral 5-FU prodrug) (Taiho Pharmaceutical Company Limited, Tokyo, Japan) was administered to control micro-lymph node metastases and distant metastases. The S-1 treatment began one month after SRS and consisted of 40-80 mg/body of S-1 for 2 weeks followed by a one week break; the treatment sequence was repeated for one year.
Table 2: Treatment Characteristics: Summary of treatment dose and treated tumor volume.

\begin{tabular}{rc}
\hline Dose (Gy) & Number of Patients (\%) \\
\hline $20-29$ & $1(4.5 \%)$ \\
\hline $30-34$ & $7(31.8 \%)$ \\
\hline $35-39$ & $10(45.5 \%)$ \\
\hline $40-42$ & $4(18.2 \%)$ \\
\hline Median (range) & $33.7(20-42)$ \\
\hline
\end{tabular}

Tumor volume $(\mathrm{cm} 3)$

\begin{tabular}{lc}
\hline Median & 24.5 \\
\hline Range & $3.4-74.4$ \\
\hline
\end{tabular}

Tumor diameter (mm)

Median $\quad 36.63$

Range

$15.21-58.65$

Following stereotactic radiosurgery, patients were monitored at either Tsurumi University Hospital or Toshiba Rinkan Hospital by oral and maxillofacial surgeons and at Yokohama CyberKnife Center by radiation oncologists. The clinical follow-up interval was every 2 weeks for the first 3 months, and every 4 weeks thereafter until the patient reached 2 years follow-up. Treatment outcome was assessed based on the Response Evaluation Criteria in Solid Tumors (RECIST) [22]. Response to treatment was evaluated using MRI at one month followup, contrast-enhanced CT at two months follow-up, PET$\mathrm{CT}$ and MRI at three months follow-up, and MRI or PETCT every three months thereafter. Toxicities were graded using the National Cancer Institute Common Toxicity Criteria Scale, Version 3.0. Overall survival after stereotactic radiosurgery was determined by Kaplan-Meier survival analysis.

\section{Results}

\section{Clinical Outcomes}

Twenty-two advanced, recurrent head and neck cancer patients were treated with SRS. In these advanced, recurrent patients treatment options were limited and the decision to treat these patients with SRS was based upon 
Table 3: Clinical Outcomes: Summary of tumor response by RESIST criteria.

\begin{tabular}{lc}
\hline $\mathbf{3 A}$ & Number of Patients (\%) \\
\hline N-patients & $9(64.3 \%)$ \\
\hline Complete Response & $1(7.1 \%)$ \\
\hline Partial Response & $1(7.1 \%)$ \\
\hline Stable Disease & $3(21.4 \%)$ \\
\hline
\end{tabular}

\section{$\mathrm{N}+$ patients}

\begin{tabular}{|c|c|c|c|c|}
\hline Clinical Stage & Complete Response & Partial Response & Stable Disease & Progressed Disease \\
\hline rTON1MO & 1 & & & \\
\hline rTONOM1 & & & 1 & \\
\hline rT2NOMO & 3 & & & \\
\hline rT3NOMO & 3 & 1 & & 1 \\
\hline rT3N1M0 & & & & 2 \\
\hline rT3N2MO & & & & 1 \\
\hline rT4NOMO & 3 & & & 2 \\
\hline rT4N1M0 & & & & 2 \\
\hline rT4N2M0 & & & & 2 \\
\hline
\end{tabular}

(A) Tumor response for patients without lymph node metastases $(\mathrm{N}-$ ) and for patients with lymph node metastases (N+). (B) Tumor response based on clinical stage.

a variety of issues including the nature of the tumor recurrence, prior treatment approaches and patient preference. Specifically, the recurrent tumors were solid masses that were well suited to a radiosurgical treatment given the proximity of critical organs. For those patients that previously received conventional radiotherapy (14/
$22)$, the ability to target the radiation dose specifically to the tumor and limit the dose to surrounding, previously irradiated, tissue was also a strong indicator for SRS. Lastly, the patients strongly preferred a treatment option that did not require hospitalization. 


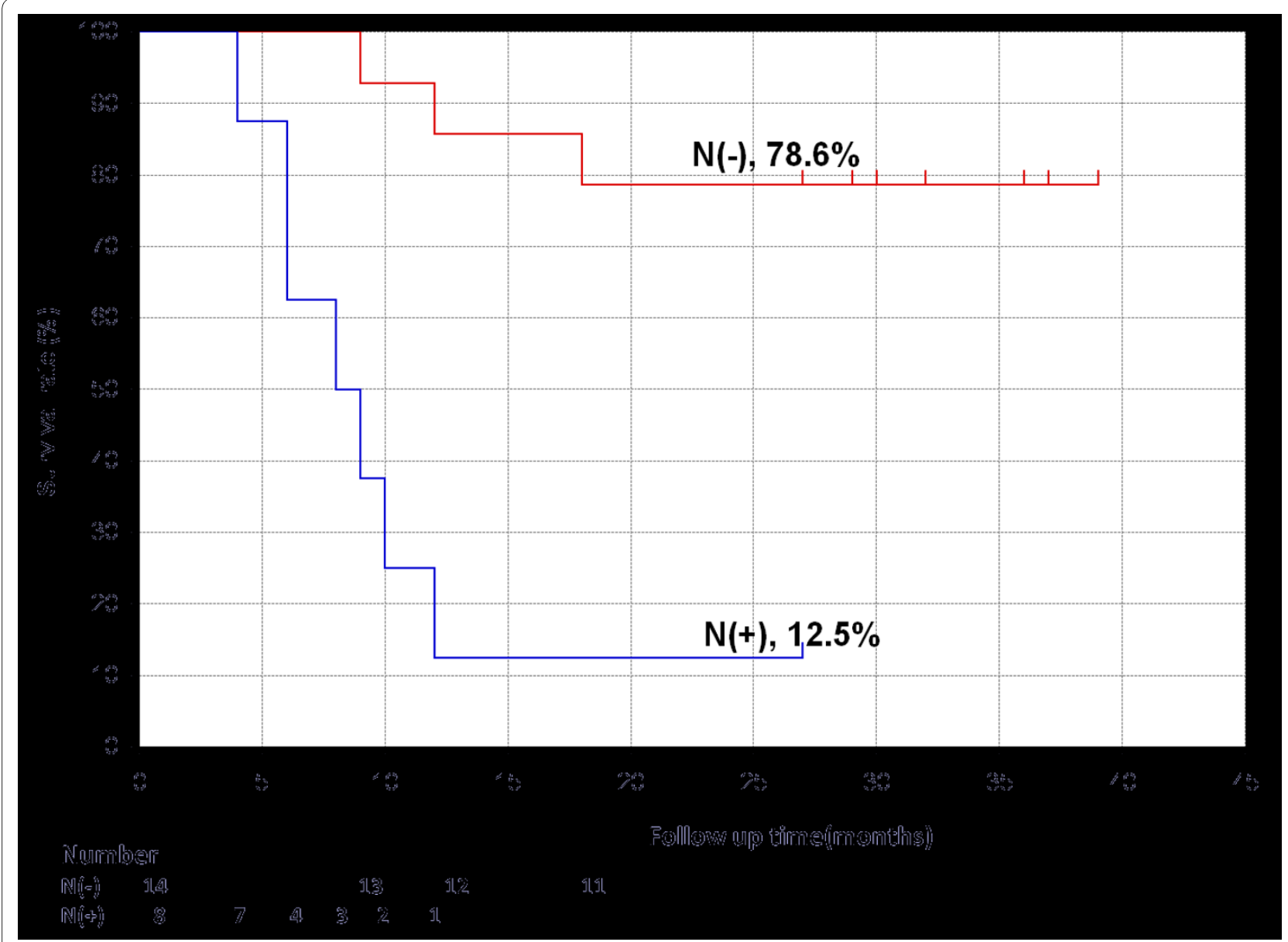

Figure 1 Kaplan-Meier plot showing the overall survival rate for patients without lymph node metastases (N-) and for patients with lymph node metastases $(\mathrm{N}+)$

The majority of patients $(12 / 22)$ received SRS treatment as outpatients with curative intent. Ten patients (45\%), however, received treatment with palliative intent while in terminal care at the hospital. At an overall median follow-up of 24 months (range, 4-39 months), 9 patients have died from their cancer. One additional patient died from acute cardiac insufficiency. For surviv-

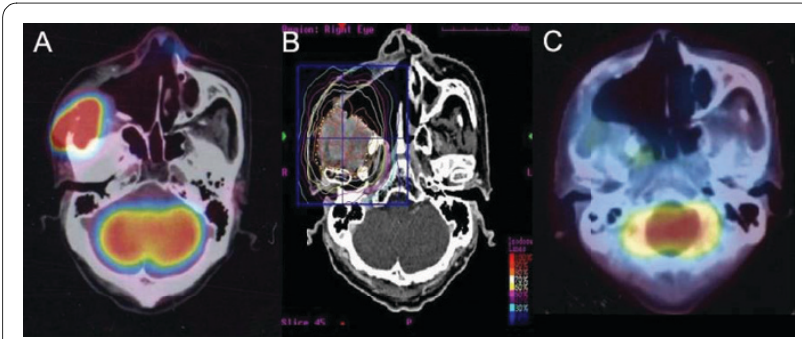

Figure 2 Case study of a 59 year old patient with a locally recurrent lesion with pterygopalatine fossa 3-years after maxillectomy. (A) Prior to treatment. (B) Treatment planning image. A total dose of 40 Gy was delivered in 5 fractions. (C) At 4-months post-treatment a complete response occurred. ing patients, the median follow-up was 32 months (range, 27-39 months).

Table A3A and Table B3B provide a summary of tumor response as assessed by RECIST criteria based on lymph node metastases and clinical stage, respectively. Specifically, for the 14 locally recurrent patients without lymph

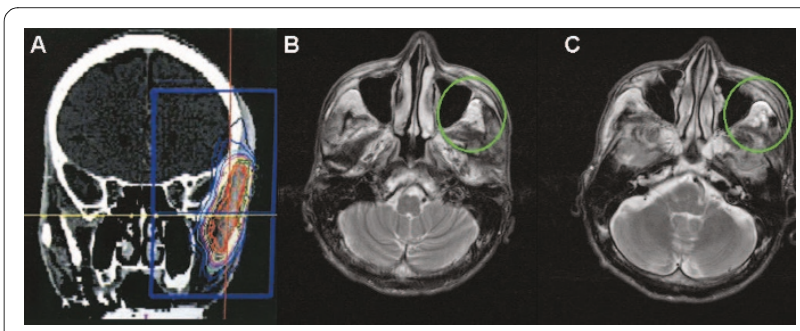

Figure 3 Case study of a T3N2cM0 patient, with tongue carcinoma that recurred as a distant metastasis in part of his temporal muscle 5 years after surgery. (A) Treatment planning image. A total dose of 30 Gy was delivered in 3 fractions. (B) Pre-treatment the recurrence is visible in his temporal muscle as denoted by the green circle. (C) At 3-months post-treatment the lesion was stable (green circle). 


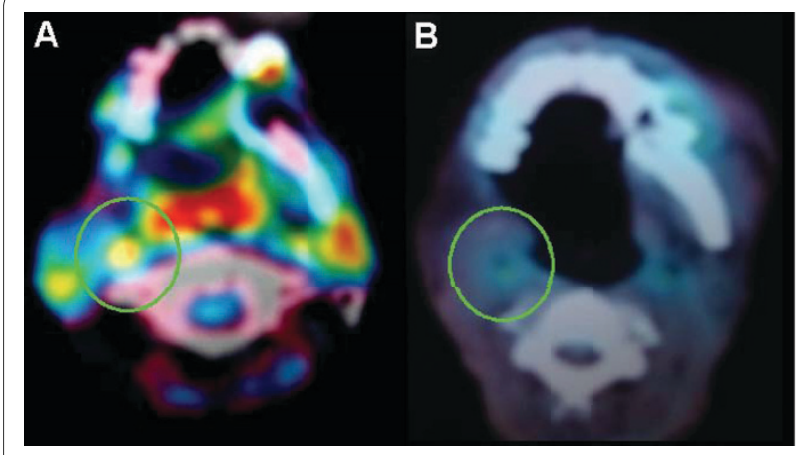

Figure 4 Case study of a patient with T3N2cM0 tongue carcinoma that recurred as a retropharyngeal lymph node metastasis 5 years after surgery. (A) Pre-treatment the lymph node metastasis is indicated by the green circle. (B) At 5-months post-treatment a complete response occurred (green circle).

node metastases, 9 patients (64.3\%) had a complete response (CR), 1 patient $(7.1 \%)$ had a partial response (PR), 1 patient (7.1\%) had stable disease (SD), and 3 patients (21.4\%) had progressive disease (PD). The three PD patients developed new lymph node metastases on the side opposite of SRS treatment. All three of these PD patients subsequently died from these late-lymph node metastases. For the 8 patients with lymph node metastases, one 1 patient, with only 1 retropharyngeal lymph node metastasis (12.5\%) had CR; the remaining 7 patients (87.5\%) all progressed. These seven patients each had 2 or 3 lymph node metastases located in their necks; upon progression they did not undergo additional treatment.

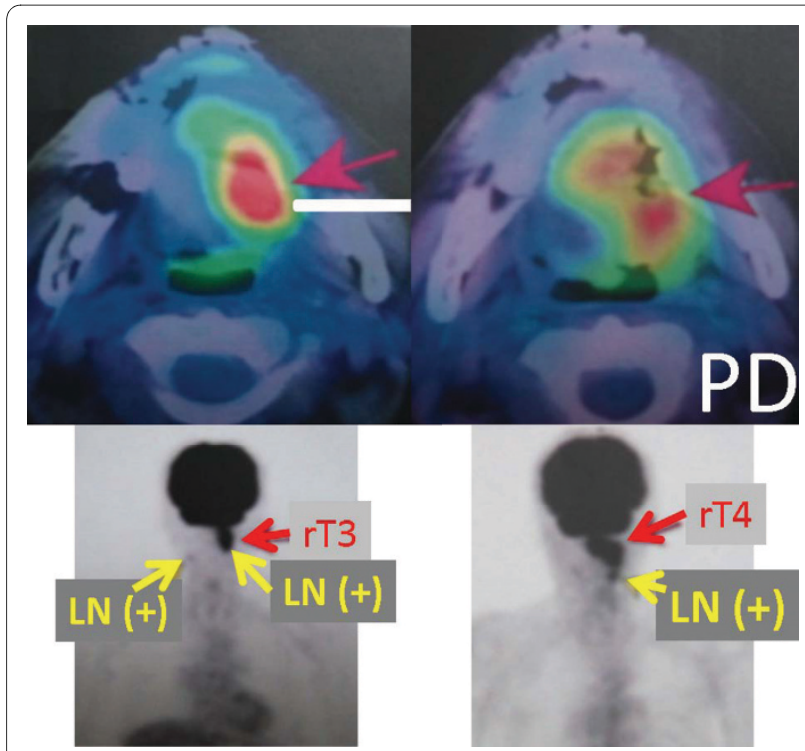

Figure 5 Case study of a patient with rT3N2cM0 tongue carcinoma that recurred 6 months after irradiation with 50 Gy in 25 fractions by conventional external beam.
Overall, at a median 2-years follow-up, 10 (45.5\%) of the 22 severe recurrent cases maintained a complete response. All 10 of these patients have returned to society and regained quality of life. The overall actuarial 2-year survival for the locally recurrent patients with and without lymph node metastases is $12.5 \%$ and $78.6 \%$, respectively (Figure 1). This difference was statistically significant with $p=0.000019$ by the log-rank test.

\section{Complications}

The first month following SRS 17 patients (77.3\%) experienced Grade 2 xerostomia and decreased taste; 5 patients (22.7\%), all of which were rT4 cases, experienced Grade 3 xerostomia and decreased taste. Of the 5 patients that experienced Grade 3 toxicity, one received prior radiation therapy (total dose $65 \mathrm{~Gy}$ ) and four received prior surgery followed by radiation therapy (total dose 50-56 Gy). After the two month follow-up, there have been no serious complications associated with the SRS re-irradiation. Fourteen of the patients who had previously received external beam radiation experienced Grade 1 (11 patients) and Grade 2 (3 patients) osteoradionecrosis at 10-18 months after SRS. None of the surgery-only patients experienced any late complications.

\section{Case reports}

Case One (Figure 2): A 59-year-old male that was found to have a locally recurrent lesion with pterygopalatine fossa 3-years after maxillectomy. SRS was delivered to a total dose of $40 \mathrm{~Gy}$ in 5 fractions. The patient experienced Grade 2 xerostomia and decreased taste without osteoradionecrosis within the first month of treatment. Four months after SRS a complete clinical response occurred. At 30 months after SRS there is no evidence of recurrence.

Case Two (Figure 3): A patient with T3N2cM0 tongue carcinoma that recurred as a distant metastasis in part of his temporal muscle 5 years after surgery. SRS was delivered to a total dose of $30 \mathrm{~Gy}$ in 3 fractions. Three months after SRS the lesion was assessed as stable and remains as stable disease at 24 months. The patient experienced hair loss at the temporal part of his head within 3 months after SRS, after which the hair grew back.

Case Three (Figure 4): A patient with T3N2cM0 tongue carcinoma that recurred with a retropharyngeal lymph node metastasis 5 years after surgery. SRS was delivered to a total dose of 23 Gy in 2 fractions. Three months after SRS the lesion exhibited a complete clinical response. At 26 months after SRS there is no evidence of recurrence. This patient was the only one of eight treated lymph node metastases in our study that had a complete response.

Case Four (Figure 5): A patient with rT3N2cM0 tongue carcinoma recurred 6 months after irradiated 
Table 4: Overview of prior stereotactic radiosurgery results for the reirradiation of recurrent head and neck carcinoma.

\begin{tabular}{|c|c|c|c|c|c|c|c|c|}
\hline Study & Patients (\#) & $\begin{array}{l}\text { SRS median } \\
\text { total dose/fx }\end{array}$ & $\begin{array}{l}\text { Follow-up } \\
\text { median, } \\
\text { range } \\
\text { (months) }\end{array}$ & $\begin{array}{l}\text { Tumor size } \\
\text { median, } \\
\text { range (cc) }\end{array}$ & $\begin{array}{c}\text { Prior } \\
\text { irradiation } \\
\text { Dose, median, } \\
\text { range (Gy) }\end{array}$ & Toxicity & $\begin{array}{c}\text { Complete } \\
\text { Response } \\
\text { Rate }\end{array}$ & Overall survival \\
\hline Voynov et al. [13] & 22 & $24 / 1-8$ & $19,11-40^{*}$ & $19.1,2.5-140.3$ & $\begin{array}{c}97.8,70.1- \\
190.3\left(\mathrm{BED}_{10}\right)\end{array}$ & No Grade 4+ & - & $22 \%$ at $2-y r s$ \\
\hline Heron et al. [15] & 25 & $25-44 / 5$ & NS & $44.8,4.2-217$ & $66-69.2$ & No Grade 3+ & $8.6 \%$ & $18 \%$ at $1-y r$ \\
\hline Roh et al. [14] & 36 & $30 / 3-5$ & 17.3 & $22.6,0.2-114.9$ & $\begin{array}{c}70.2,39.6- \\
134.4\end{array}$ & $\begin{array}{l}\text { No Grade 4+ } \\
36 \% \text { Grade } 3\end{array}$ & $42.9 \%$ & $\begin{array}{l}52.1 \% \text { at } 1-y r \\
30.9 \% \text { at } 2-y r s\end{array}$ \\
\hline Rwigema et al. [23] & 85 & $35 / 1-5$ & $6,1.3-39$ & $25.1,2.5-162$ & $70,32-170.7$ & $\begin{array}{l}\text { No Grade 4+ } \\
4.7 \% \text { Grade } 3\end{array}$ & $34 \%$ & $\begin{array}{c}48 \% \text { at } 1-y r, 16 \% \\
\text { at 2-yrs }\end{array}$ \\
\hline Sulman et al. [31] & $78^{\S}$ & IMRT 60 Gy & $25,0-81$ & $64.1,2.9-425.4$ & $60,16-75$ & $\begin{array}{l}20 \% \text { severe } \\
\text { Including } 1 \% \\
\text { Grade } 5\end{array}$ & - & $58 \%$ at $2-y r s$ \\
\hline Siddiqui et al. [25] & $44^{5} \dagger$ & $\begin{array}{l}13-18 / 1 \text { or } \\
36-48 / 5-8\end{array}$ & $6.8,1.5-48$ & $15.5,1.7-155 \ddagger$ & $63.5,50.4-74 \ddagger$ & $\begin{array}{c}6.7 \% \text { Grade 3‡ } \\
9 \% \text { Grade } 4 \neq\end{array}$ & $31 \%$ & $\begin{array}{l}38.1 \% \text { at } 1-y r \\
14.3 \% \text { at } 2-y r s\end{array}$ \\
\hline Unger et al. [24] & 65 & $30 / 2-5$ & $16^{*}$ & $75,7-276$ & $67,32-120$ & $11 \%$ Grade 4+ & $54 \%$ & $41 \%$ at $2-y r s$ \\
\hline Current Study & $22(14 \mathrm{~N}-, 8 \mathrm{~N}+)$ & $33.7 / 2-5$ & $24,4-39$ & $24.5,3.4-74.4$ & $40-65$ & $\begin{array}{c}\text { No Grade } 4+ \\
22.7 \% \text { Grade } 3\end{array}$ & $\begin{array}{c}45.4 \% \\
(\mathrm{~N}-64.3 \% \\
\mathrm{N}+12.5 \%)\end{array}$ & $\begin{array}{l}\mathrm{N}-78.6 \% \text { at } 2 \text {-yrs } \\
\mathrm{N}+12.5 \% \text { at } 2 \text {-yrs }\end{array}$ \\
\hline
\end{tabular}

* Surviving patients

† Additional non-recurrent patients treated, data presented for recurrent patients only unless otherwise noted.

₹ Includes non-recurrent patients.

$\S$ Includes patients with non-squamous cell carcinomas; data presented is for combined population.

with 50 Gy in 25 fractions by conventional external beam. SRS was delivered to a total dose of 35 Gy in 5 fractions. Three months after SRS for the recurrent lesion of the tongue and $\mathrm{N}+$ lesions, these lesions increased in size and were assessed as progressive disease. The patient experienced Grade 3 xerostomia and decreased taste as well as Grade 2 osteoradionecrosis of the mandible bone. This patient died six months after SRS as a result of a large number of lymph node metastases.

\section{Discussion}

Our results demonstrate that CyberKnife frameless stereotactic radiosurgery for patients with recurrent head and neck carcinoma is feasible and safe in the setting of previous irradiation. In the case of local recurrence without lymph node metastases, 9 out of 14 (64.3\%) patients had a complete response with a 2-year overall survival rate of $78.6 \%$. Overall, 10 out of $22(45.5 \%)$ of the advanced, recurrent patients maintain a complete response at a median 2-years follow-up.
Several stereotactic radiosurgery results, also known as fractionated stereotactic radiotherapy (fSRT), for re-irradiation of recurrent head and neck carcinomas have been reported [13-15,23-25]. The complete response rates for these studies vary from $8.6-54 \%$ with 2 -year overall survival rates ranging from $14.3-41 \%$ and 1 -year overall survival rates of $18-52.1 \%$ (see Table 4 ). In addition, one study has reported 2-year overall survival rates of $58 \%$ for reirradiation of recurrent head and neck cancer using IMRT. As the ranges of these outcomes suggest, the heterogeneity between these various studies is large. Various factors, including tumor stage, tumor volume, adequate irradiation dose, prior treatment, and anatomical site complexly, influenced these reported outcomes. In several of these studies $[13,14,23]$ patients with lymph node metastases have been included, but the reported outcomes have not been divided between those patients with and without lymph node metastases. Our reported overall survival of $78.8 \%$ for our subset of patients without lymph node involvement exceeds all of the prior published survival rates. 
Another factor affecting the reported overall survival rates is the use of chemotherapy. Recent evidence suggests that concurrent administration of chemotherapy may reduce the risk of micrometastases. A study of nasopharyngeal carcinoma demonstrated that progression-free survival among the patients who were treated with radiation alone was $24 \%$ at 3 years, compared to $69 \%$ in the combined treatment group [26]. In our study, starting one month post-SRS and continuing for one year, $100 \%(22 / 22)$ of the patients received low dose S-1 oral chemotherapy to maintain local control and to avoid lymph node and distant metastases. We choose to use S1 , an oral 5-fluorouracil (5-FU), based on several studies showing promising safety and efficacy results with this chemotherapy agent for the treatment of advanced head and neck squamous cell carcinoma [27-30]. This addition of S-1 to the SRS treatment may have also contributed to the satisfactory outcomes observed in this study.

While our results are very promising for cases without lymph node metastases, in the cases with lymph node metastases only 1 of 8 patients had a complete response and the 2 -year overall survival rate was $12.5 \%$. Since these lymph node metastases were mostly locally advanced lesions, neck dissection was not available. Given the low observed control rate, we recommend that patients eligible for surgery under general anesthesia undergo a combined salvage treatment strategy of neck dissection for the regional lymph node metastases and SRS for the locally recurrent lesion.

Reported toxicity rates for reirradiation of recurrent head and neck carcinoma include late Grade 4 and higher toxicity rates of $9 \%$ [25] and $11 \%$ [24], a rate of 36\% Grade 3 toxicity [14], and a rate of $20 \%$ severe toxicity including $1 \%$ Grade 5 toxicity for an IMRT reirradiation study [31]. In comparison, our $22.7 \%$ rate of Grade 3 toxicity with no higher grade toxicities is promising.

\section{Conclusions}

At a median 2-years follow-up, 45.5\% (10/22) of the advanced recurrent patients maintained a complete response. For the local recurrent patients with nonlymph node metastases $64.3 \%(9 / 14)$ of patients had a complete response and the 2-year actuarial overall survival rate is $78.6 \%$. Toxicity was acceptable with no observed grade 4 or higher toxicity. Hypofractionated robotic stereotactic CyberKnife radiosurgery treatment is feasible, safe, and well-tolerated for patients with local recurrence in head and neck carcinoma.

\section{Conflict of interests statement}

The authors declare that they have no competing interests.

\section{Authors' contributions}

KK and KS were responsible for the treatment of the patients and collection of data. All authors were responsible for gathering and interpreting data, manuscript revision and final manuscript approval.

\section{Author Details}

'Department of Oral and Maxillofacial Surgery, Tsurumi University, School of Dental Medicine. 2-1-3 Tsurumi, Tsurumi-ku, Yokohama, 230-8501, Japan, ${ }^{2}$ Yokohama CyberKnife Center 574-1 Ishizawacyo, Asahi-ku, Yokohama, 241 0014, Japan and ${ }^{3}$ Department of Oral and Maxillofacial Surgery, Toshiba Rinkan Hospital 7-9-1 Kamitsuruma, Sagamihara, 228-0802, Japan

Received: 3 March 2010 Accepted: 9 June 2010

Published: 9 June 2010

\section{References}

1. Chuang SC, Scelo G, Tonita JM, et al:: Risk of second primary cancer among patients with head and neck cancers: A pooled analysis of 13 cancer registries. Int J Cancer 2008, 123:2390-2396.

2. Forastiere A, Koch W, Trotti A, Sidransky D: Head and neck cancer. NEngl J Med 2001, 345:1890-1900.

3. Licitra $L$, Vermorken JB: Is there still a role for neoadjuvant chemotherapy in head and neck cancer? Ann Oncol 2004, 15:7-11

4. De Crevoisier R, Bourhis J, Eschwege F: Modified fractionated radiotherapy in head and neck squamous cell carcinoma (HNSCC) \& reirradiation in recurrent head and neck carcinomas. Cancer Treat Res 2003, 114:199-212.

5. Kramer NM, Horwitz EM, Cheng J, et al.: Toxicity and outcome analysis of patients with recurrent head and neck cancer treated with hyperfractionated split-course reirradiation and concurrent cisplatin and paclitaxel chemotherapy from two prospective phase I and II studies. Head Neck 2005, 27:406-414.

6. Spencer $S$, Wheeler R, Peters $G$, et al:: Phase 1 trial of combined chemotherapy and reirradiation for recurrent unresectable head and neck cancer. Head Neck 2003, 25:118-122.

7. Oksuz DC, Meral G, Uzel O, Cagatay P, Turkan S: Reirradiation for locally recurrent nasopharyngeal carcinoma: treatment results and prognostic factors. Int J Radiat Oncol Biol Phys 2004, 60:388-394.

8. Yu KH, Leung SF, Tung SY, et al: Survival outcome of patients with nasopharyngeal carcinoma with first local failure: a study by the Hong Kong Nasopharyngeal Carcinoma Study Group. Head Neck 2005, 27:397-405

9. Tate DJ, Adler JR Jr, Chang SD, et al.: Stereotactic radiosurgical boost following radiotherapy in primary nasopharyngeal carcinoma: impact on local control. Int J Radiat Oncol Biol Phys 1999, 45:915-921.

10. Kawaguchi K, Yamada H, Horie A, Sato K: Radiosurgical treatment of maxillary squamous cell carcinoma. Int J Oral Maxillofac Surg 2009, 38:1205-1207

11. Pai PC, Chuang CC, Wei KC, et al:: Stereotactic radiosurgery for locally recurrent nasopharyngeal carcinoma. Head Neck 2002, 24:748-753.

12. Low JS, Chua ET, Gao F, Wee JT: Stereotactic radiosurgery plus intracavitary irradiation in the salvage of nasopharyngeal carcinoma. Head Neck 2006, 28:321-329.

13. Voynov G, Heron DE, Burton S, et al:: Frameless stereotactic radiosurgery for recurrent head and neck carcinoma. Technol Cancer Res Treat 2006, 5:529-535.

14. Roh KW, Jang JS, Kim MS, et al:: Fractionated stereotactic radiotherapy as reirradiation for locally recurrent head and neck cancer. Int $J$ Radiat Oncol Biol Phys 2009, 74:1348-1355.

15. Heron DE, Ferris RL, Karamouzis M, et al:: Stereotactic Body Radiotherapy for Recurrent Squamous Cell Carcinoma of the Head and Neck: Results of a Phase I Dose-Escalation Trial. Int J Radiat Oncol Biol Phys 2009.

16. Le QT, Tate D, Koong A, et al: Improved local control with stereotactic radiosurgical boost in patients with nasopharyngeal carcinoma. Int J Radiat Oncol Biol Phys 2003, 56:1046-1054.

17. Jansen EP, Keus RB, Hilgers FJ, et al:: Does the combination of radiotherapy and debulking surgery favor survival in paranasal sinus carcinoma? Int J Radiat Oncol Biol Phys 2000, 48:27-35.

18. Fu D, Kuduvalli G: A fast, accurate, and automatic 2D-3D image registration for image-guided cranial radiosurgery. Med Phys 2008, 35:2180-2194 
19. Antypas C, Pantelis E: Performance evaluation of a CyberKnife G4 image-guided robotic stereotactic radiosurgery system. Phys Med Biol 2008, 53:4697-4718

20. Romanelli P, Schaal DW, Adler JR: Image-guided radiosurgical ablation of intra- and extra-cranial lesions. Technol Cancer Res Treat 2006, 5:421-428.

21. Hara W, Soltys SG, Gibbs IC: CyberKnife robotic radiosurgery system for tumor treatment. Expert Rev Anticancer Ther 2007, 7:1507-1515.

22. Therasse P, Arbuck SG, Eisenhauer EA, et al: New guidelines to evaluate the response to treatment in solid tumors. European Organization for Research and Treatment of Cancer, National Cancer Institute of the United States, National Cancer Institute of Canada. J Natl Cancer Inst 2000, 92:205-216.

23. Rwigema JC, Heron DE, Ferris RL, et al:: Fractionated Stereotactic Body Radiation Therapy in the Treatment of Previously-Irradiated Recurrent Head and Neck Carcinoma: Updated Report of the University of Pittsburgh Experience. Am J Clin Oncol 2009.

24. Unger KR, Lominska CE, Deeken JF, et al:: Fractionated Stereotactic Radiosurgery for Reirradiation of Head-and-Neck Cancer. Int J Radiat Oncol Biol Phys 2010 in press.

25. Siddiqui $F$, Patel M, Khan M, et al:: Stereotactic body radiation therapy for primary, recurrent, and metastatic tumors in the head-and-neck region. Int J Radiat Oncol Biol Phys 2009, 74:1047-1053.

26. Agulnik M, Epstein JB: Nasopharyngeal carcinoma: current management, future directions and dental implications. Oral Oncol 2008, 44:617-627.

27. Tsukuda M, Kida A, Fujii M, et al.: [Long-term results of S-1 administration as adjuvant chemotherapy for advanced head and neck cancer]. Gan To Kagaku Ryoho 2007, 34:1215-1225.

28. Yamashita T, Shinden S, Watabe T, Shiotani A: Outpatient chemotherapy with S-1 for recurrent head and neck cancer. Anticancer Res 2009, 29:577-581.

29. Suzuki S, Ishikawa K: Safety and efficacy of S-1 chemotherapy in recurrent/metastatic head and neck cancer. J Infect Chemother 2009, 15:335-339.

30. Kawasaki M, Watanabe $\mathrm{K}$, Watanabe J: [TS-1 and irradiation combination therapy for head and neck cancer]. Gan To Kagaku Ryoho 2006, 33:1077-1080

31. Sulman EP, Schwartz DL, Le TT, et al: IMRT reirradiation of head and neck cancer-disease control and morbidity outcomes. Int J Radiat Oncol Biol Phys 2009, 73:399-409.

doi: $10.1186 / 1748-717 X-5-51$

Cite this article as: Kawaguchi et al., Stereotactic radiosurgery may contribute to overall survival for patients with recurrent head and neck carcinoma Radiation Oncology 2010, 5:51

Submit your next manuscript to BioMed Centra and take full advantage of:

- Convenient online submission

- Thorough peer review

- No space constraints or color figure charges

- Immediate publication on acceptance

- Inclusion in PubMed, CAS, Scopus and Google Scholar

- Research which is freely available for redistribution

Submit your manuscript at www.biomedcentral.com/submit
C Biomed Central 